\section{The role of prophylaxis of bacterial infections in children with acute leukemia/non-Hodgkin lymphoma}

\author{
Elio Castagnola \\ Infectious Diseases Unit, Istituto \\ Giannina Gaslini, Genoa, Italy
}

\section{Abstract}

Infections represent a well-known complication of antineoplastic chemotherapy that may cause delay of treatment, with alteration of the antineoplastic program and dose-intensity, or even the death of a patient that could heal from his/her neoplasia. Bacterial infections are a major cause of morbidity and mortality in patients who are neutropenic following chemotherapy for malignancy. Therefore a program of antibiotic prophylaxis for febrile neutropenia may be considered in the management strategy of cancer patients.

\section{Comments}

When such a program is planned some questions have to be asked. ${ }^{1}$ The first should be which disease we want to prevent: febrile neutropenia, bacteremia or other bacterial invasive infections, or death from bacterial disease, and the efficacy of the prophylaxis against the specific disease we chosen. The frequency of the disease to prevent and the effectiveness of prophylaxis will condition the number need to treat (NNT), i.e. the number of patients that will receive prophylaxis to prevent 1 of the even we want to avoid. The lower the NNT, the higher is the probability that prophylaxis will be effective (i.e. low NNT yes prophylaxis, high NNT no prophylaxis). The NNT depends on frequency of the disease in the controls, consequences of the treatments (efficacy $v$ toxicity and/or drug interactions), costs (of prophylaxis and the treatment of the disease if not prevented), and selection of resistances and there is no standardization to decide if the NNT is satisfactory, i.e. in a disease with highly severe consequences also a high NNT could be acceptable. ${ }^{2}$ Many clinical trials have demonstrated the efficacy of different antibiotics (mainly cotrimoxazole, ciprofloxacin, amoxicillin-clavulanate) as prophylaxis of febrile neutropenia both in children and adults with leukemia, ${ }^{3-6}$ with different NNT according to the type of disease that should be prevented: the lowest for fever, the highest for death. ${ }^{5}$
However in the absolute majority of these studies patients were enrolled only once (for statistical reasons) and the effectiveness of prophylaxis during repeated neutropenic periods has been studied only in one, very questionable clinical trial in adults solid tumors (by the way the effect of prophylaxis was could be observed only during the neutropenic period following the first cycle of chemotherapy, but not in the subsequent ones). 7,8 Therefore the effectiveness of prophylaxis during repeated neutropenic periods (i.e. the normal course of antineoplastic chemotherapy) must still be demonstrated, especially in leukemic patients. In this sense, the analysis of epidemiological data in neutropenic children with cancer shows that the risk of repeated febrile episodes during subsequent neutropenic periods is influenced by the aggressiveness of chemotherapy in acute leukemia/non-Hodgkin lymphoma and the level of neutropenia at onset in solid tumors, with no effect estimated for antibacterial prophylaxis in both cases. ${ }^{9}$

The second question to be answered planning a prophylactic program is if the even we want to prevent it a severe one (Yes: prophylaxis, No: no prophylaxis), and, if it could be easily treated in case of occurrence. The most frequent infectious event in neutropenic children is fever of unknown origin, which occurs in near $50 \%$ of neutropenic periods following aggressive treatment for acute leukemia/nonHodgkin lymphoma or conditioning regimen for hemopoietic stem cell transplant, and in less than 1/3 of neutropenic periods following less aggressive chemotherapy for acute leukemia/non-Hodgkin lymphoma or any type of treatment for solid tumors. ${ }^{10}$ Gram-negative bacteremia that is the most severe (and feared) complications and one of the major targets of prophylaxis is diagnosed in no more than $15 \%$ of episodes of febrile neutropenia in children. ${ }^{10}$ The majority of episodes of febrile neutropenia is treated quite easy, with a reasonably low rate of failure (i.e. the need to changes the initial treatment treatment or mortality) and the most recent pediatric guidelines indicates monotherapy with a beta-lactam active against Pseudomonas as the most reasonable choice for initial empirical treatment. ${ }^{11}$

However, in presence of infection due to resistant strains the risk of failure increases, with a significant reduction of patients' survival.12-14 Selection of resistant strains is probably the worst adverse event induced by widespread use of antibacterial prophylaxis in neutropenic cancer patients. ${ }^{14-17}$ In spite of many inferences from clinical trials and meta analyses suggest that administration of prophylaxis had no impact on he selection of resistant bacteria, $3,5,18$ the search for colonization by resistant strains is generally absent in randomized clinical trials on antibacterial prophylaxis of
Correspondence: Elio Castagnola, Infectious Diseases Unit, Istituto Giannina Gaslini, Largo G. Gaslini 5, 16147 Genoa, Italy.

Tel.: +39.010.5636.429 - Fax: +39.010 .3763436$

Email: eliocastagnola@ospedale-gaslini.ge.it

Key words: acute leukemia, non-Hodgkin lymphoma, bacterial infections, prophylaxis.

Conflict of interests: the author declares no potential conflict of interests.

Received for publication: 30 January 2014.

Accepted for publication: 28 February 2014.

This work is licensed under a Creative Commons Attribution NonCommercial 3.0 License (CC BYNC 3.0).

CCopyright E. Castagnola, 2014

Licensee PAGEPress, Italy

Pediatric Reports 2014; 6:5332

doi:10.4081/pr.2014.5332

febrile neutropenia. Indeed, only in one recent randomized clinical trial of antibiotic prophylaxis for febrile neutropenia in children the risk of selection of resistant strains during antibacterial prophylaxis was addressed in a satisfactory way with evaluation of intestinal flora colonizing the patients before and after the administration of prophylaxis. ${ }^{6}$

In general, only epidemiological studies analyzing bacterial infections/colonization when a prophylactic program is adopted in a specific patients' population may give a correct evaluation of the risk of selecting resistant bacteria induced by antibiotic prophylaxis. In this sense, data from a pediatric epidemiological study clearly show that the widespread use of antibacterial prophylaxis causes ecological changes, with acquisition of resistant pathogens also in patients who did not receive antibiotics for preventing febrile neutropenia. ${ }^{19}$

As specifically regards antibacterial prophylaxis of febrile neutropenia in children with acute leukemia/non-Hodgkin lymphoma, only 2 randomized, double-blind, placebo-controlled clinical trials have been performed in this setting so far. ${ }^{4,6}$ In the first one, the administration of amoxicillin-clavulanate significantly reduced, $-21 \%$, the incidence of fever in neutropenic children with acute leukemia/lymphoma, while no effect could be demonstrated in solid tumors. In the second one performed in acute leukemia, administration of ciprofloxacin determined a $23 \%$ reduction of febrile episodes compared with placebo. In both cases the NNT to prevent one episode of febrile neutropenia was very similar, 5 in the first and 4 in the second (no NNT could be calculated for bacteremia or death since the studies were not planned to analyze the effect of 
prophylaxis on these complications, but it is presumably very high for both conditions). 5

What becomes apparent from both studies is that for every 100 neutropenic patients receiving prophylaxis, $75-80$ of them are treated unnecessarily to prevent the remaining 20-25 from developing during and neutropenia. If we consider that the incidence of Gram-negative bacteremia, the most feared complication because of high mortality, generally represents no more than $10-15 \%$ of all febrile neutropenic episodes we estimate that we would administer unnecessarily prophylaxis in $96-97$ patients to prevent Gram-negative bacteremia in 3-4 subjects. This number could still be considered as acceptable, if antibiotic resistance was not an emerging problem. This specific problem was not addressed in the first pediatric trial, while in the second it was observed that after two weeks from the end of prophylaxis $95 \%$ of patients receiving ciprofloxacin were colonized by resistant Enterobacteriaceae, while this proportion was $27 \%$ in patients treated with placebo. International guidelines now recommend the implementation of systematic surveillance for monitoring rates of resistance among Gram-negatives and in some cases it is recommended that prophylaxis should be abandoned because probably ineffective, when resistance rate is greater than $20 \% .11,20$

In the period 2004-2010 a program of antibacterial prophylaxis with oral amoxicillinclavulanate or intravenous ampicillin-sulbactam for prevention of fever after aggressive treatment for acute leukemia/non-Hodgkin lymphoma or during the pre-engraftment period in HSCT was implemented at the Giannina Gaslini Institute, Genoa, Italy. This strategy was associated with a strict monitoring of the etiology and antimicrobial susceptibility of pathogens isolated from blood cultures that showed an increase of infections due to ceftazidime-resistant, not ESBL-producing Gramnegatives (that was detected in more than $30 \%$ of Gram-negatives isolated from blood cultures, and perhaps due to the production of the beta-lactamase amp-C induced by amoxicillinclavulanate) and an increase of resistance to fluoroquinolone that resulted $17 \%$ of Gramnegatives causing bacteremias, ${ }^{21}$ even if this drug was never used for prophylaxis. As a consequence antibacterial prophylaxis was interrupted in 2010 for children with acute leukemia/lymphoma, while it was maintained during the pre-engraftment period of stem cell transplant (both autologous and allogeneic) that represent a very peculiar period at risk, since antibacterial prophylaxis has been shown effective in a single period of administration (i.e. the single period of prophylaxis administered in a randomized clinical trial).

Stemming from all these observations, i.e. relatively low frequency of febrile neutropenia and Gram-negative bacteremia, absence of effect during repeated neutropenic periods and increasing in severe infections due to antibiotic-resistant strains, it is my personal opinion that now is the time when antibacterial prophylaxis in neutropenic children with cancer should be abandoned at least during chemotherapeutic regimens.

\section{References}

1. McQuay HJ, Moore RA. Issues involved in making choices in prophylaxis. Ann Intern Med 1997;126:712-20.

2. Sinclair JC, Cook RJ, Guyatt JH, et al. When should an effective treatment be used? Derivation of the threshold number needed to treat and the minimum event rate for treatment. J Clin Epidemiol 2001; 54:253-62.

3. Bucaneve G, Micozzi A, Menichetti F, et al. Levofloxacin to prevent bacterial infection in patients with cancer and neutropenia. $\mathrm{N}$ Engl J Med 2005;353:977-87.

4. Castagnola E, Boni L, Giacchino M, et al. A multicenter, randomized, double blind placebo-controlled trial of amoxicillin/clavulanate for the prophylaxis of fever and infection in neutropenic children with cancer. Pediatr Infect Dis J 2003;22:359-65.

5. Gafter-Gvili A, Fraser A, Paul M, et al. Antibiotic prophylaxis for bacterial infections in afebrile neutropenic patients following chemotherapy. Cochrane Database Syst Rev 2012;1:CD994386.

6. Laoprasopwattana K, Khwanna T, Suwankeeree P, et al. Ciprofloxacin reduces occurrence of fever in children with acute leukemia who develop neutropenia during chemotherapy. Pediatr Infect Dis J 2013;32:e94-8.

7. Cullen M, Steven N, Billingham L, et al. Antibacterial prophylaxis after chemotherapy for solid tumors and lymphomas. N Engl J Med 2005;353:988-98.

8. Cullen M, Billingham L, Gaunt C, Steven $\mathrm{N}$. Rational selection of patients for antibacterial prophylaxis following chemotherapy. J Clin Oncol 2007;25:4821-8.

9. Bagnasco F, Haupt R, Fontana V, et al. Risk of repeated febrile episodes during chemotherapy-induced granulocytopenia in children with cancer: a prospective single center study. J Chemother 2012;24:155-60.

10. Castagnola E, Fontana V, Caviglia I, et al. A prospective study on the epidemiology of febrile episodes during chemotherapyinduced neutropenia in children with cancer or after hemopoietic stem cell transplantation. Clin Infect Dis 2007;45:1296-304.

11. Lehrnbecher T, Phillips R, Alexander S, et al. Guidelines for the management of fever and neutropenia in children with cancer and/or undergoing hematopoietic stem cell transplantation. J Clin Oncol 2012;30: 4427-38.

12. Caselli D, Cesaro S, Ziino 0, et al. Multidrug resistant Pseudomonas aeruginosa infection in children undergoing chemotherapy and hematopoietic stem cell transplantation. Haematologica 2010; 95:1612-5.

13. Trecarichi EM, Tumbarello M, Caira M, et al. Multidrug resistant Pseudomonas aeruginosa bloodstream infection in adult patients with hematologic malignancies. Haematologica 2011;96:e1-3.

14. Kang CI, Kim SH, Park WB, et al. Bloodstream infections caused by antibiotic-resistant gram-negative bacilli: risk factors for mortality and impact of inappropriate initial antimicrobial therapy on outcome. Antimicrob Agents Chemother 2005; 49:760-6.

15. Kern WV, Steib-Bauert M, de With K, et al. Fluoroquinolone consumption and resistance in haematology-oncology patients: ecological analysis in two university hospitals 1999-2002. J Antimicrob Chemother 2005;55:57-60.

16. Reuter S, Kern WV, Sigge A, et al. Impact of fluoroquinolone prophylaxis on reduced infection-related mortality among patients with neutropenia and hematologic malignancies. Clin Infect Dis 2005;40:1087-93.

17. Ng ES1, Liew Y, Koh LP, Hsu LY.. Fluoroquinolone prophylaxis against febrile neutropenia in areas with high fluoroquinolone resistance: an Asian perspective. J Formos Med Assoc 2010;109: 624-31.

18. Leibovici L, Paul M, Cullen M, et al. Antibiotic prophylaxis in neutropenic patients: new evidence, practical decisions. Cancer 2006;107:1743-51.

19. Castagnola E, Haupt R, Micozzi A, et al. Differences in the proportions of fluoroquinolone-resistant Gram-negative bacteria isolated from bacteraemic children with cancer in two Italian centres. Clin Microbiol Infect 2005;11:505-7.

20. Freifeld AG, Bow EJ, Sepkowitz KA, et al. Clinical practice guideline for the use of antimicrobial agents in neutropenic patients with cancer: 2010 update by the Infectious Diseases Society of America. Clin Infect Dis 2011;52:e56-93.

21. Castagnola E, Moroni C, Bandettini R, et al. Ciprofloxacin prophylaxis in children with acute leukemia. What should we do in an era of increasing antibiotic resistance? Pediatr Infect Dis J 2013;32:581. 